PROFESSOR GEOFFREY L DICKENS (Orcid ID : 0000-0002-8862-1527)

Article type : Original Article

Manuscript category: Empirical research - quantitative

\title{
Harm-reduction approaches for self-cutting in inpatient mental health settings: Development and preliminary validation of the Attitudes to Self-cutting Management (ASc- Me) Scale
}

Running Head: Attitudes to self-cutting management

Leah HOSIE RMN BSc(Hons), Senior Staff Nurse, NHS Tayside

Geoffrey L. DICKENS* RMN MA PhD, Professor Mental Health Nursing, Abertay

University, Bell Street, Dundee, UK, DD1 1HG.

*Corresponding author.

Author GLD is now Professor Mental Health Nursing, Western Sydney University and South West Sydney Local Health District, Locked Bag 7103 Liverpool BC NSW 1871, Australia Email: g.dickens@westernsydney.edu.au

Conflict of interest statement: The authors have no conflicts of interest to declare

Funding: This work was supported by NHS Tayside who funded author LH's Masters by Research Degree

This article has been accepted for publication and undergone full peer review but has not been through the copyediting, typesetting, pagination and proofreading process, which may lead to differences between this version and the Version of Record. Please cite this article as doi: 10.1111/jpm.12498

This article is protected by copyright. All rights reserved. 


\section{Author statement:}

Study conception and design: Dickens

Acquisition of data: Hosie

Analysis and interpretation of data: Hosie, Dickens

Drafting of manuscript: Hosie, Dickens

Critical revision: Dickens, Hosie

Both authors give final approval to the submitted version

The funder played no role in the conception, design, data analysis, manuscript preparation, or in the decision to publish.

The authors have no competing interests to declare

Abbreviations used in this article are: Attitudes to Self-cutting Management Scale [ASc-Me]; Self Harm Inventory [SHI]; Attitudes to Deliberate Self-Harm Questionnaire [ADSHQ]; SelfHarm Antipathy Scale [SHAS ]; National Institute for Health and Clinical Excellence [NICE]; Principal Components Analysis [PCA]; Intraclass Correlation Coefficient [ICC]

\section{ABSTRACT}

Introduction: Harm-reduction approaches for self-harm in mental health settings have been under-researched.

Aim: To develop a measure of the acceptability of management approaches for self-cutting in mental health inpatient settings.

Methods: Stage one: scale items were generated from relevant literature and staff/service user consultation. Stage two: A cross-sectional survey and statistical methods from classical test theory informed scale development. 
Results/Findings: At stage one $N=27$ staff and service users participated. At stage two $N=215$ people ( $n=175$ current mental health practitioners and $n=40$ people with experience of self-cutting as a UK mental health inpatient) completed surveys. Principal components analysis revealed a simple factor structure such that each method had a unique acceptability profile. Reliability, construct validity, and internal consistency were acceptable. The harmreduction approaches 'advising on wound-care' and 'providing a first aid kit' were broadly endorsed; 'providing sterile razors' and 'maintaining a supportive nursing presence during cutting' were less acceptable but more so than seclusion and restraint.

Discussion: The Attitudes to Self-cutting Management scale is a reliable and valid measure that could inform service design and development.

Implications for practice: Nurses should discuss different options for management of selfcutting with service users. Harm reduction approaches may be more acceptable than coercive measures.

Keywords: Attitude; Harm reduction; Inpatients; Self-injurious behaviour; Surveys and questionnaires

\section{Accessible summary}

\section{What is known on the subject}

- Nurses in mental health inpatient settings use a range of methods to try and help service users who self-harm

- Harm reduction approaches are intended to help service users reduce the impact of their self-harm rather than simply to prevent them self-harming

- Harm reduction techniques might be helpful for people who cut themselves because there are some clear ways harm can be minimised such as providing advice about cutting

- No one has previously tried to measure whether harm reduction techniques are more or less acceptable to mental health practitioners and service users than traditional methods. 


\section{What the paper adds to existing knowledge}

- The paper describes the development of the Attitudes to Self-cutting Management scale. It met the criteria required of a good measurement tool.

- Each method of managing self-cutting has a unique acceptability profile

- Harm reduction methods like advising on wound care and providing a first aid kit are endorsed by nurses and former service users

- Nurses providing sterile razors or remaining present during self-cutting attract more divergent opinions but are preferred to seclusion and restraint

\section{What are the implications for practice}

- Nurses should talk through the approaches to management with service users and agree which techniques are preferred.

\section{Relevance statement}

Self-harm is common in inpatient mental health settings and nurses are required to deliver care and management. Harm reduction approaches have become common in relation to care in the fields of intravenous drug use and sexual health. There has been some qualitative work around harm reduction approaches in relation to self-harm. Use of such approaches is inconsistent and views on them have only been investigated qualitatively. The development of a measure to look quantitatively at the issue of self-cutting specifically is described. Self-cutting was selected since it seems to be the most appropriate form of selfharm to use in a harm reduction model. The resulting tool has clear relevance for further research and for practice. Notably it shows that different management methods have their own acceptability profile which could inform, nurse-service users discussions about care.

\section{INTRODUCTION}

Self-harm or self-injury, 'the deliberate destruction of body tissue without conscious intent of suicide' (Fontaine, 2003: p.221), is the cause of considerable financial expense and health-related concern in society more widely, and in mental health services specifically.

Estimates of lifetime rates of self-harm in the general population globally lie between $3.5 \%$ and 6.5\% (Briere \& Gil, 1998; Information Centre for Health and Social Care, 2007; Meltzer et al., 2002; Swannell et al., 2014). Those who self-harm, and especially those who do so repeatedly, add significantly to the financial burden on society due to increased costs in relation to medical and psychiatric service use (Sinclair et al., 2010; Tsiachristas et al., 2014).

This article is protected by copyright. All rights reserved. 
The issue is particularly acute in psychiatric settings where, across 25 studies, $17.4 \%$ (range $0.7-68.8 \%$ ) of patients have self-harmed (James et al., 2012); and up to 21\%, specifically by self-cutting (Briere \& Gil, 1998). Nursing staff working on mental health wards use a variety of strategies to prevent or respond to self-harm, but there is little research about the effectiveness of any of them (James et al., 2012). Nurses appear to be dissatisfied with their own practice, perceiving an over-emphasis on medication-based approaches (O’Donovan \& Gijbels, 2006); difficulty in understanding self-harming behaviour (Wilstrand et al., 2007); problems in striking the right balances between safety maintenance, protection of dignity, and appropriate relational boundaries (James et al., 2007; O’Donovan, 2007; Wilstrand et al., 2007).

Research about the use of different containment measures for the management of a range of conflict behaviours in the inpatient setting, including self-harm as well as aggression, absconding, and rule-breaking, has identified that nurses tend to hold attitudes towards different containment measures rather than towards containment in general (Dack et al., 2012). The measures investigated, however, do not include specific harm-minimisation approaches for the management of self-injury; nor, with the exception of management of aggression and violence (e.g., Duxbury, 2003), do they measure attitudes about the management of specific categories of behaviour. This may be considered unsurprising in relation to self-harm, since harm-minimisation approaches in this sphere tend to be behaviour-specific rather than generic and adaptable across conflict-related scenarios. For example, such approaches can involve practices including providing advice on woundhygiene, or supplying sterile cutting blades, activities which have no direct corollaries in terms of the management of, say, aggression. Nevertheless, there has been a growing interest in harm-minimisation approaches for self-injury, and such techniques have been advocated by some who suggest that a more baldly preventative approach can be distressing, 
stigmatising, detrimental to the therapeutic relationship with professionals, and can contribute to behavioural escalation (Duperouzel \& Fish, 2008; Holley et al., 2012; Lindgren et al., 2011; Pembroke, 1994; Shaw, 2012). Much debate around the use of harm-minimisation approaches has been rooted in non-empirical discussions of ethics and legalities, much of whose tone suggests a stark binary choice between adopting or proscribing such techniques (e.g., Edwards \& Hewitt, 2011; Gutridge, 2010; Sullivan, 2017). In the UK, official guidance is to recommend 'tentative approaches to harm reduction for some people who self-harm in the community' (National Institute for Health and Clinical Excellence [NICE], 2011: p.259. Current authors' italics).

Little research has investigated harm-minimisation approaches in the inpatient mental health setting. A recent survey and qualitative interview study conducted with UK wardbased mental health practitioners (James et al., 2017) found some dichotomised attitudes: the majority of participants did not believe people should be allowed to self-harm in a safe environment, nor that they had a right to self-harm in any event. Interview data also revealed considerable uncertainty around assessment and management of people using a harmreduction approach, and dissonance between the approach and their own moral beliefs and understanding of related legal issues. However, it is not known where harm-minimisation approaches stand relative to other methods of self-harm management in terms of their acceptability either for mental health practitioners or service users.

\section{Rationale}

Ascertaining the level of support for these approaches relative to a range of other techniques for self-cutting management, therefore, holds considerable potential for informing future education and practice. A number of scales have been developed to measure the attitudes of healthcare professionals towards self-harm, including the Attitudes to Deliberate Self-Harm Questionnaire (ADSHQ; McAllister et al., 2002), the Self-Harm Antipathy Scale (SHAS; Patterson et al., 2007a) and the Self Harm Inventory (SHI; Sansone \& Sansone, 2010). Each considers self-cutting as one of a range of self-harming methods, but none focuses on it as a discrete behaviour. While this is not problematic per se, it may become an issue in the context of harm-minimisation approaches to self-harm management, particularly 
in the inpatient setting where breaking of the skin is the most common self-harm behaviour (James et al., 2017). In addition, while there has been some discussion in the literature about promoting what, some argue, are safer alternatives to self-harm including taking a bath that is hotter or colder than usual, snapping rubber bands on the wrists, or using a toothbrush on the skin (Pengelly et al., 2008), self-cutting is, to the best of our knowledge, the only form of actual self-harm (i.e., destroying bodily tissue) which has been nominated as a candidate for use in a harm-minimisation. context. Potential harm-minimisation techniques could involve advising on the use of sterile implements, educating on safer parts of the body to cut (Pengelly et al., 2008), supplying sterile razors, or even remaining present during a cutting event to offer support. It is very difficult to imagine any other method (e.g., self-poisoning, ligature, immolation, firearms) being nominated similarly; indeed, such an approach to the use of pharmaceuticals is explicitly ruled out due to there being 'no safe limits' (e.g., NICE, 2004).

\section{Aim and objectives}

The aim of the current study was to develop a measure to investigate the attitudes of inpatient mental health service staff and service users towards the management of self-cutting within those settings. Specific objectives were to i) base the measure on contemporary research evidence and best practice; ii) involve relevant stakeholders in the development process; iii) base development on sound principles of relevant measurement theory; iv) use the tool to investigate the acceptability of using harm minimisation methods in the inpatient setting relative to other methods; v) to investigate mental health nursing staff versus service user differences in terms of the acceptability of self-cutting management measures.

\section{METHODS}

Study reporting was informed by the COnsensus-

based Standards for the selection of health Measurement INstruments (COSMIN) guidelines

(Mokkink et al., 2012).

This article is protected by copyright. All rights reserved. 


\section{Design}

A two-stage mixed-methods design was utilised to investigate nursing staff's and service users' attitudes towards the management of self-cutting in inpatient mental health settings. Stage one comprised tool development and involved generation and refinement of potential scale items through literature and policy review, and a consultation exercise with mental health service user representatives and mental health nursing professionals. Stage two comprised testing of the tool and analysis for potential reduction of items and utilized a crosssectional survey and analysis techniques from classical test theory to develop valid and reliable measures to investigate the construct (Urbina, 2004).

\section{Ethical approval and conduct}

The project was approved by the Abertay University Research Ethics Committee and NHS Tayside Research \& Development Department (Project Reference Number:

SHS_R_2015-16_11 $7^{\text {th }}$ December 2015). At stage one both staff and service user participants were provided with full written details of the study and gave written consent. At stage two potential participants were again provided with full written details; on this occasion consent was taken to be implied by return or completion of the questionnaire. At both stages all participants were provided with details of possible sources of support in the event of any distress. Participants were also offered the option of contacting either of the researchers (both experienced mental health nurses).

\section{Setting and participants}

At stage-one mental health nursing staff from the lecturing staff and post-registration student nurse community at Abertay University were recruited. Additionally, mental health service users were recruited from one service user support group in one NHS Board in

This article is protected by copyright. All rights reserved. 
Scotland. Investigator LH obtained permission to attend the group in order to gain feedback on the draft tool.

At stage-two, inclusion criteria for mental health staff participants were registered mental nurses, nursing and healthcare assistants, and student mental health nurses. The sample was opportunistic, comprising self-selecting respondents. Nursing staff participants were drawn from two NHS Health Boards in Scotland via a paper-based survey, and from UK-wide via a web-based survey (Survey Monkey ${ }^{\mathrm{TM}}$ ), a link to which was circulated via fora including the Mental Health Nurse Academics UK (https://mhnauk.wordpress.com/) mailing list. This is a grouping with representatives from every university providing pre-registration mental health nurse training in the UK. Potential staff participants were eligible if they had recent experience of working in a mental health inpatient ward in which self-harm by cutting had occurred. People self-identifying as having prior experience as a UK mental health inpatient and having been subject to care and management interventions for self-cutting during their stay were recruited via online support forums (National Self-Harm Network [http://www.nshn.co.uk/] and Recover Your Life [http://www.recoveryourlife.com/]) and through a monthly e-bulletin produced by the organisation Self-Injury Support (https://www.selfinjurysupport.org.uk/). Potential participants were provided with a link to the web-based survey. Inclusion criteria was self-identifying as having personal experience of self-cutting during a mental health inpatient admission.

Calculating the required sample size in scale development studies can be problematic since the adequacy of the sample size cannot be determined until after the data have been analysed (Henson \& Roberts, 2006). Researchers have followed a number of recommendations based on heuristics about the number of participants that are required per scale item e.g., a minimum participant: item ratio of 10:1, or the minimum number in total (e.g., $N=300$; Worthington Whittaker, 2006). Since each of the 18 management techniques 
comprised six items it was necessary to ensure a sample size sufficient for a 108-item tool. This suggested an absolute minimum of $N=300$; however, Cabrera-Nguyen's (2010) guidelines for reporting on exploratory factor analysis studies advises to run preliminary analyses of communality to determine if further data collection is required. Suggested criteria are that if (a) communalities are $>0.50$ or there are 10:1 items with factor loadings of roughly .4 then a sample size of 150 to 200 is likely to be adequate; further, if communalities are all at least 00.60 then even smaller sample sizes may suffice (Worthington Whittaker, 2006).

\section{Measures}

\section{Demographic details}

Demographic information was collected via a purpose-designed schedule (see Table 2 for details).

\section{Attitudes to Self-cutting Management Scale (ASc-Me)}

The aim was to develop a tool to specifically measure attitudes towards the full range of approaches to the care and management of inpatients who display self-cutting behaviour including verbalisations of intention to self-cut, cutting-related cognition, and actual cutting. The initial item pool was drawn from NICE (2013) guidelines, and from the wider literature. Eighteen management techniques were included in total (see Table 1).

In the final version of the tool, respondents rate each management method in terms of its effectiveness (e.g., 'This method is effective'), acceptability, respectfulness, safety for staff, safety for the patient, and their preparedness to use (staff)/ their preparedness to be 
subject to (service users) the technique on a 5-point Likert scale ('Strongly Agree' [High score] to 'Strongly Disagree' [Low score]). Respondents also indicate whether they have been subjected to the method (patients) or have utilised it (staff). The order of item presentation was randomised.

\section{Attitudes to Containment Measures Questionnaire (ACMQ; Bowers et al, 2004)}

The ACMQ is an 11-item tool that measures attitudes towards commonly used containment measures used to manage conflict on mental health wards. Respondents rate their agreement with the acceptability of each method on a 5-point Likert scale, and indicate whether they have used the technique (staff version) or been subject to it (service user version). In the original validation version of the ACMQ respondents were asked to rate each method in respect of its acceptability, effectiveness and so on in a similar manner to the AScMe scale described above. The tool is an 11-factor tool, each method representing its own factor. Later versions of the ACMQ have simply asked respondents about 'acceptability'; since the validation study found high communalities within ACMQ factors - in effect, ratings of acceptability, effectiveness and so on are highly inter-related for each containment method

- it was deemed acceptable to reduce measurement for each factor to a proxy item ('acceptability'). In the current study we used the 11-item amended version based on the assumption that the tool has received sufficient validation. Since items are proxy scores for factors we therefore could not calculate Cronbach's alpha statistics for the ACMQ. We examined relationships between the five ACMQ and ASc-Me items which address attitudes to similar interventions (increased observations; PRN; seclusion; forced intramuscular medication; and physical restraint) albeit within the differing contexts of self-cutting specifically (ASc-Me) and conflict more widely including physical and verbal aggression (ACMQ).

This article is protected by copyright. All rights reserved. 


\section{Self-Harm Antipathy Scale (SHAS; Patterson et al., 2007).}

The SHAS is a 30-item tool for measuring health care professionals' attitudes towards self-harm covering six factors: competence appraisal, care futility, client intent manipulation, acceptance and understanding, rights and responsibilities, and needs function. Response is on a 7-point Likert scale and the whole scale has good internal consistency $(\alpha=0.89)$ (Patterson et al., 2007). Since we aimed to measure the attitudes of both nursing staff and service users we removed items from the 'care futility' and 'competence appraisal' factors since they were not relevant for service users. Following omissions, 17 statements remained.

\section{Procedure}

During stage one an initial pool of items reflecting a wide range of management techniques for self-cutting in the inpatient environment was generated along with a brief description for each from policy documents and the wider literature. A method of rating and scoring items was also devised (5-point likert scale rated from 'Strongly Disagree' to 'Strongly Agree'). The initial tool formed the basis of data collection at stage one. Mental health professionals were asked to complete the schedule and provide written feedback about readability, feasibility, and acceptability of the items. The same staff were also asked to complete the schedule on a second occasion in order to establish inter-rater reliability. Service user respondents were asked to provide verbal feedback about the schedule. Completed questionnaires were not used in the subsequent study and neither of these groups were targeted for recruitment into the main study. All feedback was then used to inform refinement of the scale.

This article is protected by copyright. All rights reserved. 
At stage two the refined scale was distributed to mental health practitioners via paper copies and through online academic and clinical mailing lists, and to people who may have self-harmed by cutting during an inpatient stay item via relevant online discussion forums (see Setting and Participants). The potential for reducing the item set through use of Principal Components Analysis and calculation of internal reliability (Cronbach's alpha) was considered. Next, indicators of construct validity, namely convergent and divergent validity, were examined to determine whether results from the new scale were congruent with measures of related constructs. Indicators of test-retest reliability - the extent to which a subsample of respondents provide similar responses over relatively brief period of time were also calculated.

\section{Data analysis plan}

All data was entered into IBM SPSS V23.0 for analysis. Descriptive statistics were calculated to examine proportions and frequencies. Additional testing is described below.

\section{Tests of data quality, distribution, stability, and scaling assumptions}

All data were examined for quality (\% missing data by item) and normality of distribution; data was within acceptable boundaries of skewness and kurtosis suggested by Hair et al. (2017). For the ASc-Me scale, descriptive and correlational analyses were conducted to evaluate scaling assumptions (e.g., similar item mean scores and variances, scores which span the entire measurement continuum, and the magnitude and similarity of corrected item-total correlations). Further analysis of scale-to-sample targeting (score means and standard deviation [SD]; floor and ceiling effects), and internal consistency (Cronbach's alpha $[\alpha])$ were conducted. 


\section{Test-retest reliability}

For ASc-Me Likert scale items, intraclass correlation coefficients (ICC) were calculated. ICCs fall between -1 and 1 with 1 (perfect negative and positive correlation respectively). ICCs are the most suitable statistic for determining the reliability of ordinal data and kappa for categorical data (Hallgren, 2012). ICCs of 0.71-0.79 are fair, 0.80-0.89 good and >0.90 excellent (Cichetti,2004). For the dichotomised 'yes'/'no' responses, the Kappa $(\mathrm{K})$ reliability coefficient was calculated; $\mathrm{K}=0.40-0.59$ is fair, $0.60-0.74$ good and 0.75-1.00 excellent (Cichetti, 2004).

\section{Principal Components Analysis of the amended SHAS and of the ASc-Me}

Principal components analysis (PCA) serves to reduce the number of variables required to adequately capture complex, multivariate constructs by identification of scale items that best represent underlying latent variables. PCA also facilitates identification of factors within the multivariate construct i.e., clusters of variables which represent meaningful and clinically useful subgroups of items. Since items had been removed from the SHAS, a PCA to determine whether the underlying structure remained valid was conducted. PCA of the ASc-Me scale was also conducted in order to explore its structure. Kaiser-Mayer-Olkin measures of sampling adequacy were conducted to test adequacy of the data for PCA; scores $\geq 0.90$ are 'excellent' while scores $<0.50$ are 'unacceptable' (Field, 2005). Bartlett's test of sphericity was conducted to measure the assumption of equal variance. The number of factors extracted following both analyses' initial solution was set for those with Eigenvalues $>1$.

Varimax rotation with Kaiser normalisation was appropriate in both analyses and was used to maximise loadings in subsequent iterations. The most satisfactory component structures were those which captured the most variance in the data, and which comprised three or more items 
all loading $>0.50$ on a single factor (i.e., no cross-loading). The internal reliability of the whole scales and their components was measured using Cronbach's alpha. George and Mallery (2003, p.231) suggest alphas $>0.9$ excellent, $>0.8$ good, $>0.7$ acceptable, $>0.6$ questionable, $>0.5$ poor, and $<0.5$ unacceptable.

\section{Construct validity}

It was anticipated that ratings for specific ASc-Me methods of management for selfcutting would be positively correlated with the single rating for its counterpart ACMQ method for containment use in general (i.e., seclusion, restraint, forced IM medication, PRN medication, observations). In contrast, it was expected that ASc-Me methods with no direct corollaries in the management of containment methods in the ACMQ (e.g., distraction, harm reduction methods) would not have significant relationships with ACMQ methods.

Specific items on the SHAS (e.g., 'people should be allowed to self-harm in a safe environment'; 'an individual has the right to self-harm') closely align with ASc-Me harm reduction techniques and therefore some significant relationships would be expected. However, given the removal of items (described in 'Measures' above) to make it relevant to service user respondents, judgement was reserved on how to proceed in terms of making hypotheses about specific relationships between aspects of the SHAS and the ASc-Me scale until the results of the PCA described above were known. Spearman's rho correlations are appropriate for use in ordinal data sets; rho falls in the range -1 to 1 ; values of $0.20-0.39$, 0.40-0.59, 0.60-0.79, and 0.80-1.0 indicate weak, moderate, strong, and very strong relationships respectively. (Weir, 2017).

This article is protected by copyright. All rights reserved. 


\section{Nursing staff/ Service user approval ratings for individual management techniques}

Finally, total approval rating scores for each technique were calculated by summing the six individual ratings. Total scores of nursing staff and service user participants were compared using independent samples t-tests.

\section{RESULTS}

\section{Scale development and test-retest reliability}

At stage-one, $N=20$ mental health nurses first provided feedback and completed the resulting amended scale on two occasions. Mean (95\% CI) ICCs of all ordinal ASc-Me items was fair at 0.70 (range 0.66 to $0.74, \mathrm{p}<0.05)$. All dichotomised items had a good degree of reliability (Mean $[95 \% \mathrm{CI}] \mathrm{K}=0.74[0.65-0.84], \mathrm{p}<0.02)$. The service user support group was attended by seven people. Responses from both groups led to minor changes in the terminology used, and expansion of the description of each management intervention, but not to addition or subtraction of items.

\section{Tests of ASc-Me data quality, distribution, stability, and scaling assumptions}

At stage two, 175 mental health nursing staff completed questionnaires (see Table 2). Thirty-eight staff requested a paper copy of the questionnaire and all (100\%) were returned. The remaining 137 (78.3\%) staff participants completed an online version of the questionnaire; the completion rate for this was $65 \%$ (35\% of individuals who accessed the questionnaire online did not go on to complete it). All 40 of the service user respondents completed the online version; the completion rate was $42 \%$ (58\% of individuals who accessed the questionnaire failed to complete it). Therefore, the total number of persons accessing the online study questionnaire was 315 , with $n=177$ completing the full battery (completion rate of 62.6\%). Incomplete online surveys were deleted listwise. This is 
acceptable when it leads to loss of minimal data: of service user non-completers all but one completed either zero or one item only $(0-0.6 \%)$ with the exception completing 18 items 10.8\%). For online nursing staff respondents, the number of missing items ranged from 125 to $165(75.3 \%$ to $99.4 \%)$; just 10 non-completers provided more than $2(1.2 \%)$ responses. Further, there were no significant differences between nurse completers and non-completers who provided information on age, gender, occupation, or experience and none between service user completers and non-completers on age, gender, or time spent in hospital. The final ASc-Me scale dataset comprised 23,220 data points (215 participants x 108 items); less than $0.1 \%$ of data was missing. Missing data was dealt with via median imputation which is acceptable when only a small amount of scores are missing (Zhang, 2016). Preliminary analysis of the communalities from the Principal Components Analysis revealed that all 108 were in excess of 0.6 (mean $=.83, S D=0.06$, range 0.62 to 0.93 ) and thus suggested that the sample size was more than adequate for further principal components analysis.

All ASc-Me data fell within acceptable levels for skewness and kurtosis. Examination of Likert scale data for all six ratings on the 18 management intervention-types revealed use of the whole scale (scores ranging from 1 to 5 ) for $88 / 108(81.5 \%)$ items; 13 of the 18 (72.2\%) management interventions contained no scale which did not use the whole range of

scores. The measurement issue with the remaining items was related to capturing disapproval of interventions related to care planning, suggesting passive distractions, and therapeutic interventions. Only for seven related items $(6.5 \%)$ did the scale fail to capture any level of disapproval at all (i.e., range 1-3 or less).

\section{Use of and exposure to self-cutting management methods}

The proportion of staff who had used each management technique ranged from $1.1 \%$ ('deliver inappropriate medical treatment') to $99.1 \%$ ('provide therapeutic interventions'). Of the most restrictive methods, seclusion (47.4\%) was reportedly used by more nurses than 
restraint (33.7\%) and forced intramuscular medication (32.6\%). With regards to harm minimisation methods, $6.3 \%$ of staff respondents had provided a razor to an inpatient and $14.3 \%$ had remained present to offer support during a self-cutting episode.

Exposure to each technique for service users ranged from $7.5 \%$ ('provide razor') to 82.5\% (passive distractions' and 'active distractions'). Exposure to the most restrictive measures was $50.0 \%$ (seclusion), $45.0 \%$ (restraint), and $42.5 \%$ (forced intramuscular medication). $15.0 \%$ had experienced 'staff present to offer support during cutting'. While $4.0 \%$ of staff said they had refused treatment as a management strategy, $65.0 \%$ of service users said they had been refused treatment. Note that totals add up to more than $100 \%$ since service users reported exposure to, and staff use of, multiple management methods.

\section{Principal components analysis of the ASc-Me}

The Kaiser-Meyer-Olkin measure of sampling adequacy statistic was adequate $(0.72)$ whilst Bartlett's test of sphericity was highly significant $(\mathrm{p}<0.001)$ indicating that principal components analysis was appropriate in this case. The 108 item-ratings for the effectiveness, acceptability, respectfulness, safety for staff, safety for the patient, and preparedness to use/be subject to the method loaded on to 17 factors. The 12 item ratings for the methods 'increasing observations to special' and 'increasing observations to close' loaded onto one factor. Cronbach's alpha scores for the item-ratings of these two methods were $\alpha=0.91$ and $\alpha=0.93$ respectively and therefore the former item was removed from the scale. The remaining 102-items loaded onto 17 -factors each comprising the six related item ratings for the technique (see Table 4). Total variance explained by the 17 -factors was $80.1 \%$. Sixteen factors displayed 'excellent' internal reliability while one was only 'good' (Intermittent observations $\alpha=0.8) . M(S D)$ Cronbach's alpha for all the included methods was $0.94(0.04)$.

This article is protected by copyright. All rights reserved. 


\section{Construct validity}

ASc-Me relationships with ACMQ. It was anticipated that five specific ASc-Me

items would be significantly positively correlated with five corresponding ACMQ items since they both describe similar management strategies (increase observations; forced intramuscular medication; seclusion; PRN; physical restraint), albeit for use in different contexts. Spearman's Rho correlations for approval of pairs of similar methods ranged from 0.33 to 0.42 . All correlations were statistically significant at the $P<0.001$ level.

\section{Principal Components Analysis of the amended SHAS and relationships with the}

$\boldsymbol{A S c}-\boldsymbol{M e}$. The Kaiser-Meyer-Olkin measure of sampling adequacy statistic (0.82) and Bartlett's test of sphericity $(\mathrm{p}<0.001)$ indicated that principal components analysis was appropriate. Analysis revealed an 11-item, 3-factor structure (see Table 3). Six items were discarded because they failed to meet selection criteria. Item groupings were agreed to reflect areas relating to i) a perceived manipulative functionality of self-harm $(\alpha=0.87 ; 26.3 \%$ of the variance); ii) a perceived positive functionality of self-harm $(\alpha=0.73 ; 18.8 \%$ of the variance); and iii) freedom of choice in relation to self-harm $(\alpha=0.70 ; 17.7 \%$ of the variance). Thus total variance explained by the three factors was $62.9 \%$.

Following inspection of the SHAS principal components analysis it was hypothesised that the latent variables 'perceived functionality' and 'freedom of choice' would correlate positively with harm minimization approaches to self-cutting ('provide advice on wound care', 'provide first aid kit', 'provide razor', 'be present during a self-cutting event') since they are intuitively congruent with attitudes that support cutting as functional and as a free choice. It was hypothesised that the remaining SHAS factor ('perceived manipulation') would not be significantly positively associated with the same items. Table 5 shows that seven of 
the eight predicted correlations (i.e. all but SHAS 'perceived functionality' and ASc-Me 'remain present during cutting event') were significant. Non-significant correlations between the SHAS 'perceived manipulation' scale and ASc-Me harm reduction items were as expected in three of four cases (i.e., all but for that between 'perceived 'manipulation and 'provide razor for cutting').

\section{Staff-service user mean scores on ASc-Me factors}

The overall rankings derived from staff and service user item-ratings were highly similar. An intraclass correlation coefficient (ICC) derived from overall rankings $=0.93$ (95\% CI 0.81, 0.97, d.f.=16, $P<0.001)$ falling in the 'excellent' range (Cicchetti, 2004) (See Table 6). In terms of magnitude of total approval ratings, there were statistically significant differences (large and medium effect sizes) on 10 of the 17 management techniques (see Table 6). For factors differing significantly, in all but one case ('refuse treatment') the difference was such that nurses approved more of the technique. There was no difference between the groups on factor total scores for 'remain present during cutting' or 'provide sterile razor for cutting'.

\section{DISCUSSION}

Discussion and debate around harm minimisation approaches for the management of self-harm date at least to NICE (2004) guidelines, yet research as recent as that by James et al (2017) has found widely inconsistent approaches in inpatient mental health settings, with nurses expressing dissatisfaction with medication-focused approaches to management yet relatively little uptake of harm minimisation approaches. Most recently, Sullivan (2017) has concluded that healthcare professionals should sometimes allow patients in mental health units to self-harm. The arguments echo those made by others including service users who say 
that approaches that aim to restrict self-injurious behavior may simply make the problem worse by disempowering people who often have a history of trauma and abuse (e.g. Shannon, 2013). With the very recent exception of work by James et al (2017) most investigative work on the issue of harm minimization has focused on ethical aspects (Sullivan, 2017) or on narrative accounts of individuals or groups of experts by experience (e.g., Baker et al., 2013). While valuable, these accounts have not attempted to investigate quantitatively the attitudes of people towards the management of self-harm approaches in inpatient mental health settings. Examples from parallel fields, such as studies of attitudes towards containment measures for conflict more generally (Bowers et al., 2007a, b; Whittington et al., 2009; MuirCochrane, et al., 2009), have demonstrated the value of such an approach since they starkly demonstrate the mismatch between attitudes towards and actual use of highly restrictive containment measures.

In this context, the ASc-Me Scale was developed primarily to aid better understanding of the attitudes of nursing staff and service users about harm-reduction approaches to selfcutting management in inpatient settings; though as a by-product the current study has investigated attitudes to a far wider range of management approaches. The ASc-Me has been developed from comprehensive published NICE (2013) guidance on techniques to manage self-harm and with user and professional involvement. The comprehensiveness, and thus the content validity, of the included techniques was underscored by the fact that no additional techniques were added in the latter consultation. The ASc-Me scale has demonstrated good preliminary psychometric properties: principal components analysis demonstrated close alignment between rating aspects for each individual technique suggesting a unique profile of acceptability for each. Test-retest reliability was acceptable, individual items were congruent with conceptually similar measures from the ACMQ and the SHAS and diverged from less 
similar ones. The 17 factors included in the final version of the tool explained in excess of $80 \%$ of measured variance.

As might be expected, different harm-reduction methods varied in terms of their acceptability; the most contentious - providing a sterile razor for self-cutting, and remaining with the patient during self-cutting - were ranked eleventh and twelfth of seventeen in terms of acceptability. Mean item score on the six acceptability items for these techniques was 3.1, representing an overall position of 'neither agree nor disagree'. All of the techniques that were ranked less acceptable than this were the most intrusive coercive containment measures available in UK psychiatric inpatient settings, or were clearly inappropriate methods of management that have no rightful place in nursing care (providing inappropriate care or refusing care). Importantly, however, the more intrusive coercive measures - notably physical restraint and forced IM medication - were even less preferred. The clear message is that the more contentious harm reduction methods do seem to be more acceptable overall to nursing staff or ex-service users who self-identify as having cut themselves during a previous mental health inpatient admission than coercive measures. Nevertheless, deep ambivalence is suggested by the significant number of nurses who either disagreed $(30 ; 17.1 \%)$ or strongly disagreed $(12 ; 6.9 \%)$ that remaining with a service user during a cutting event was acceptable. By far the largest proportion of nurses were 'uncertain' $(86 ; 49.1 \%)$, suggesting strongly that that this is an area where educators, clinical leaders, and regulators should provide space for discussion and critical reflection. This reflects and extends previously highlighted findings of dichotomization among the mental health nursing workforce on this issue (James et al., 2017).

This article is protected by copyright. All rights reserved. 
While the similarity of nursing staffs' and service users' ranking of acceptability of each technique was considerable there were significant differences relating to the magnitude of that approval for well over half of the factors. Interestingly, there was no difference on the groups' ratings of the potentially controversial harm reduction techniques of maintaining a nursing presence during a cutting episode and providing a sterile razor for cutting. Overall, however, nursing staff appeared to overvalue a range of self-cutting management techniques relative to service users for both coercive interventions (seclusion, physical restraint) and less overtly intrusive approaches (therapeutic interventions, care planning, passive and active distractions, wound advice, making other staff aware and providing a first aid kit). Nurses should be aware that their relative therapeutic optimism may outstrip that of service users; at the same time their relative appreciativeness of seclusion and restraint should not blinker them from exploring the use of a full range of harm reduction methods.

One consideration to be made is about precisely who should be consulted about major changes to current practice. For example, during development of the ACMQ (Bowers et al., 2004) efforts were made to recruit large and representative samples of staff and current inpatients resident on acute psychiatric wards to gauge their opinion. This seems appropriate since aggression and violence, the main reason for coercive measures, is visible to and can affect all patients, staff and the therapeutic environment. The current authors, of course, welcome further testing on a wider scale of the ASc-Me, but also raise the question about whether the wider inpatient population should have such significant input into the development of policies and practices that may only be relevant to a few. Further, since the harm minimisation interventions in question are less obtrusive than coercive measures and are, self-evidently, designed for use in private as opposed to in public, these authors strongly believe that primacy should be given to the views of those whom such a policy would affect: patients who self-cut and nurses who would be required to manage the intervention. 


\section{Implications for practice and education}

Mental health nurses working in acute inpatient settings should consider offering a full range of harm-minimisation techniques. They should develop appropriate protocols and policies for their use. While all the techniques might not be appropriate at all times, it should be recognised that providing sterile razor or staying with a patient during cutting may well be preferable for that individual then seclusion or restraint. This highlights the need for nurses to discuss the options with patients/service users in order to ascertain their feelings and preferences, and to provide information. All other non-coercive and non-inappropriate approaches were broadly supported by both surveyed groups and should be offered as standard. It should be noted, however, that, where differences exist, they unanimously reveal lower acceptability for service users than nursing staff.

Current harm-reduction practice in the UK is inconsistent (James et al., 2017). The current study strengthens the case for a more coherent approach supported by policy, education, and training. The current study represents preliminary development only of a measure of management acceptability and much more intensive further work is required on particular models for delivering these techniques in practice. This would require, for example, further validation of the ASc-Me with a larger sample of both nurses and service users.

\section{Limitations}

The current sample was a self-selecting convenience sample and results may therefore not necessarily reflect the views of the wider nursing, or self-cutting service-user, population. This is especially relevant to the results comparing the two groups completing the current 
survey. A full $80 \%$ of staff-side respondents were staff nurses; this suggests despite our recruitment being somewhat pushed through an academic mechanism (MHNAUK) it did filter to the 'shop floor'. Educational level was not investigated so we do not know if nurserespondents were more engaged nurses on, for example, post-registration courses then a representative sample might be. Similarly, service users may have been more active-types who were engaged in internet and real-world fora who were made aware of the survey. Larger scale surveys with more representative samples of service users who self-harm are therefore required to further validate the tool.

\section{Conclusion}

Rightly, much thought has gone into the ethics and legalities of harm-reduction approaches to the management of self-injury -and specifically self-cutting - in the inpatient mental health environment. However, a lack of data on attitudes towards this contentious issue, particular in terms of the acceptability of harm minimization approaches relative to other approaches, and the relative acceptability of specific harm-reduction approaches to one another, has held back the debate. Drawing on approaches to the measurement of attitudes to containment a valid tool for measuring a range of approaches towards self-cutting management has been developed. Further research is required to ensure samples of both staff and service users are representative.

\section{REFERENCES}

Baker, C. Shaw, C. \& Biley, F. (Eds). Our Encounters with Self-Harm. Monmouth, UK: PCCS Books.

Bowers, L., Alexander, J., Simpson, A., Ryan, C., \& Carr-Walker, P. (2004). Cultures of psychiatry and the professional socialization process: the case of containment methods for disturbed patients. Nurse Education Today, 435-442. DOI: 10.1016/j.nedt.2004.04.008

Bowers, L., Simpson, A., Alexander, J., Ryan, C. \& Carr-Walker, P. (2007) Student psychiatric nurses' approval of containment measures: relationship to perception of 
aggression and attitudes to personality disorder. International Journal of Nursing Studies, 44(3), 349-356.

Bowers, L., van der Werf, B., Vokkolainen, A. Muir-Cochrane, E., Allan, T. \& Alexander, J. (2007). International variation in attitudes to containment measures for disturbed psychiatric inpatients. International Journal of Nursing Studies, 44(3), 357-364.

Cabrera-Nguyen, P. (2010). Author guidelines for reporting scale development and validation results. Journal of the Society for Social Work and Research, 1(2), 99-103. DOI: 10.5243/jsswr.2010.8

Cichetti, D.V. (2004). Guidelines, criteria, and rules of thumb for evaluating normed and standardized assessment instruments in psychology. Psychological Assessment, 6, 284-290.

Comey, A.L. \& Lee, H.B. (1992). A First Course in Factor Analysis. Lawrence Erlbaum, Hillside, NJ.

Dack, C., Ross, J., \& Bowers, L. (2012). The relationship between attitudes towards different containment measures and their usage in a national sample of psychiatric inpatients. Journal of Psychiatric and Mental Health Nursing, 19, 577-586. DOI: 10.1111/j.1365-

2850.2011.01832.x

Duperouzel, H. and Fish, R. (2008). Why couldn't I stop her? Self-injury: the views of staff and clients in a medium secure unit. British Journal of Learning Disabilities, 361, 59-65.

DOI: $10.1111 / \mathrm{j} .1468-3156.2007 .00486 . \mathrm{x}$

Duxbury, J. (2003). Testing a new tool: The Management of Aggression and Violence Attitude Scale (MAVAS). Nurse Researcher, 10, 39-52. DOI: 10.7748/nr2003.07.10.4.39.c5906

Edwards, S.D. and Hewitt, J. (2011). Can supervising self-harm be part of ethical nursing practice? Nursing Ethics, 18, 79-87. DOI: 10.1177/0969733010386166

Field, A.P. (2005). Discovering Statistics Using SPSS. 2nd ed. London: Sage.

George, D. \& Mallery, P. (2003). SPSS for Windows Step by Step: A Simple Guide and Reference (11.0 update). 4th ed. Boston, MA: Allyn \& Bacon.

Gutridge, K. (2010). Safer self-injury or assisted self-harm? Theoretical Medicine and Bioethics. 31, 79-92. DOI: 10.1007/s11017-010-9135-Z

Hair, J.F., Hult, G.T.M., Ringle, C.M., \& Sarstedt, M. (2017). A Primer on Partial Least Squares Structural Equation Modeling (PLS-SEM). 2nd Ed. Thousand Oaks, CA: Sage.

Hallgren, K. A. (2012). Computing inter-rater reliability for observational data: An overview and tutorial. Tutorials in Quantitative Methods for Psychology, 8(1), 23-34.

Henson, R., \& Roberts, J. (2006). Use of exploratory factor analysis in published research: Common errors and some comment on improved practice. Educational and Psychological Measurement, 66, 393-416. DOI:10.1177/0013164405282485

Holley, C., Horton, R., Cartmail, L., \& Bradley, E. (2012). Self-injury and harm minimisation on acute wards. Nursing Standard, May 23-29;26(38):50-56. DOI:

10.7748/ns2012.05.26.38.51.c9113

James, K. Samuels, I., Moran, P. \& Stewart, D. (2017). Harm reduction as a strategy for supporting people who self-harm on mental health wards: The views and experiences of practitioners. Journal of Affective Disorders, 214, 67-73. DOI: 10.1016/j.jad.2017.03.002

This article is protected by copyright. All rights reserved. 
James, K., Stewart, D., \& Bowers, L. (2012). Self-harm and attempted suicide within inpatient psychiatric services: A review of the literature. International Journal of Mental Health Nursing, 21, 301-309. DOI: 10.1111/j.1447-0349.2011.00794.x

Lindgren, B-M., Öster, I., Åström, S., and Graneheim, U.H. (2011). 'They don't understand. You cut yourself in order to live.' Interpretative repertoires jointly constructing interactions between adult women who self-harm and professional caregivers. International Journal of Qualitative Studies on Health and Well-being, 63. DOI: 10.3402/qhw.v6i3.7254

McAllister, M., Creedy, D., Moyle, W., \& Farrugia, C. (2002). Nurses' attitudes towards clients who self-harm. Journal of Advanced Nursing, 40, 578-586. DOI: 10.1046/j.13652648.2002.02412.

Mokkink, L.B., Terwee, C.B., Patrick, D.L., Alonso, J., Stratford, P.W., Knol, D.L., Bouter, L.M., \& de Vet, H.C.W. (2012). COSMIN Checklist Manual. Leiden, Netherlands: Institute for Health and Care Research. Accessed $20^{\text {th }}$ July 2018 at: http://www.cosmin.nl/images/upload/files/COSMIN\%20checklist\%20manual\%20v9.pdf

Muir-Cochrane, E., Bowers, L., \& Jeffery, D. (2009). Comparison in attitudes between nursing and other students in relation to containment measures for disturbed psychiatric patients. Nurse Education Today, 29(1), 83-90.

National Institute for Clinical Excellence (2004). Self-harm. The short-term physical and psychological management and secondary prevention of self-harm in primary and secondary care. Summary of management and treatment. London: NICE.

National Institute for Health and Clinical Excellence (2011). Self-harm: Longer Term Management. Clinical Guideline CG133. http://www.nice.org.uk/guidance/CG133

National Institute of Health and Clinical Evidence (NICE). (2013). Self-harm: Quality standard [QS34]. London: National Institute for Health and Care Excellence. Accessed 03/01/2018: www.nice.org.uk/guidance/qs34

O'Donovan, A. (2007). Pragmatism rules: The intervention and prevention strategies used by psychiatric nurses working with non-suicidal self-harming individuals. Journal of Psychiatric and Mental Health Nursing, 14, 64-71. DOI: 10.1111/j.1365-2850.2007.01044.x

O'Donovan, A. \& Gijbels, H. (2006). Understanding psychiatric nursing care with nonsuicidal self-harming patients in acute psychiatric admission units: The views of psychiatric nurses. Archives of Psychiatric Nursing, 20, 186-192. DOI: https://doi.org/10.1016/j.apnu.2005.12.007

Patterson, P., Whittington, R. \& Bogg, J. (2007). Measuring nurse attitudes towards deliberate self-harm: The Self-Harm Antipathy Scale (SHAS). Journal of Psychiatric and Mental Health Nursing, 14, 438-445. DOI: 10.1111/j.1365-2850.2007.01102.x

Pembroke, L. (1994). Self-Harm: Perspectives from Personal Experience. Chipmunk Publishing, London.

Pengelly, N., Ford, B., Blenkiron, \& Reilly, S. (2008). Harm minimisation after repeated selfharm: development of a trust handbook. Psychiatric Bulletin, 32, 60-63. DOI: 10.1192 / pb. bp. 106.012070

Sansone, R.A. \& Sansone, L.A. (2010). Measuring self-harm behaviour with the self-harm inventory. Psychiatry (Edgemont), 7, 16-20.

Shaw, C. (2012). Harm-minimisation for self-harm. Mental Health Today, 19-21.

This article is protected by copyright. All rights reserved. 
Shannon, M.T. (2013). For girls like me. In: C. Baker, C. Shaw, \& F. Biley (Eds). Our Encounters with Self-Harm. Monmouth, UK: PCCS Books.

Sinclair, J.M.A., Gray, A., Rivero-Arias, Saunders, K.E.A. \& Hawton, K. (2011). Healthcare and social services resource use and costs of self-harm patients. Social Psychiatry \& Psychiatric Epidemiology, 46, 263-271. DOI: https://doi.org/10.1007/s00127-010-0183-5

Sullivan, P.J. (2017). Should healthcare professionals sometimes allow harm? The case of self-injury. Journal of Medical Ethics, 43, 319-323. DOI:

http://dx.doi.org/10.1136/medethics-2015-103146

Sullivan. G.M. \& Feinn, R. (2012). Using effect size - or why the p value is not enough. Journal of Graduate Medical Education, 4. DOI:10.4300/JGME-D-12-00156.1

Swannell, S.V., Martin, G.E., Page, A., Hasking, P., \& St John, N.J. (2014). Prevalence of nonsuicidal self-injury in nonclinical samples: systematic review, meta-analysis and metaregression. Suicide and Life Threatening Behaviour, 44, 273-303. DOI: 10.1111/sltb.12070

Tsiachristas, A., McDaid, D., Casey, D., Brand, F., Leal, J., Park, A-L., Geulayov, G., Hawton, K. (2017). General hospital costs in England of medical and psychiatric care for patients who self-harm: A retrospective analysis. The Lancet Psychiatry, 4, 759-767. DOI: https://doi.org/10.1016/S2215-0366(17)30367-X

Urbina S. (2004). Essentials of Psychological Testing. New Jersey, NJ: John Wiley \& Sons, Inc.

Weir, A. (2017). Spearman's correlation. Accessed 03/01/18:

http://www.statstutor.ac.uk/resources/uploaded/spearmans.pdf

Wilstrand, C., Lindgren, B. M., Gilje, F. \& Olofsson, B. (2007). Being burdened and balancing boundaries: A qualitative study of nurses' experiences caring for patients who selfharm. Journal of Psychiatric and Mental Health Nursing, 14, 72-78. DOI: 10.1111/j.13652850.2007.01045.x

Whittington, R. Bowers, L. Nolan, P. Simpson A., and Neil, L. (2009) Approval ratings of inpatient coercive interventions in a national sample of mental health service users and staff in England. Psychiatric Services, 60(6), 792-798.

Worthington, R. \& Whittaker, T. (2006). Scale development research: A content analysis and recommendations for best practices. Counseling Psychologist, 34, 806-838.

DOI: $10.1177 / 0011000006288127$

Zhang, Z. (2016). Missing data imputation: focusing on single imputation. Annals of Translational Medicine, 4(1), 9. DOI: 10.3978/j.issn.2305-5839.2015.12.38

This article is protected by copyright. All rights reserved. 
Table 1: ASc-Me Scale initial items following mental health nursing staff and service user consultation

1.Passive distractions [Suggest and provide indirect alternatives to cutting - distraction techniques, relaxation techniques, engaging in unrelated activities]

2.Active distractions [Suggest/ provide pinging elastic bands, using ice cubes, drawing on self with red pen, toothbrush on skin]

3.Care planning [Develop a person-cent red, individualised care plan and risk assessment to address cutting] 4.Therapeutic interventions [Provide psychological interventions aimed at self-harm reduction e.g. problem solving approaches, consideration of self-cutting function]

5.Wound care hygiene [Provide advice on wound care, cleaning, and signs of infection]

6.Make others aware [Make all nursing staff aware that a person is self-cutting or feels like self-cutting]

7.First Aid kit [Provision of a First Aid kit containing dressings, etc. for immediate wound care]

8.Sterile self-cutting equipment [Provide sterile cutting implements - razors or scalpels - to lower risk of infection following a cutting event]

9.Maintain nursing presence [Nurse remains present during self-cutting event to provide support and ensure safety]

10.Offer PRN [Administration of PRN medication - oral medication given with patient consent]

11.Intermittent observation [Increase observations so patient is checked on at predetermined intermittent times by staff e.g. every 10 minutes]

12.Close observation - in sight of [Increase observations so patient remains in sight of a member of nursing staff at all times]

13. Close observation - at arm's length [Increase observations so patient remains within arm's length of a member of nursing staff at all times]

14.Seclusion [Moving and isolating patient within their room or another specified area of the ward] 15.Inappropriate treatment [e.g., stitch wounds without anaesthetic as a disincentive to self-cutting] 16.Refuse treatment [ Refuse to provide treatment to the individual who engages in self-cutting] 17.Physical restraint [Prevent patient moving/self-cutting by nursing staff using specific manual holding techniques]

18.Forced IM medication [Forced Intra Muscular medication - injection of intramuscular medication (e.g. sedatives) given without consent 
Gender

$\begin{array}{lcc}\text { Male } & 40(22.9) & 1(2.5) \\ \text { Female } & 135(77.1) & 39(97.5)\end{array}$

Age (Years)

$\begin{array}{lll}\leq 19 & 1(0.6) & 2(5) \\ 20-29 & 37(21.1) & 17(42.5) \\ 30-39 & 35(20.0) & 16(40.0) \\ 40-49 & 55(31.4) & 3(7.5) \\ 50-59 & 43(24.6) & 2(5.0) \\ 60+ & 4(2.3) & 0(0.0)\end{array}$

Employment

Student nurse

29 (16.6)

Healthcare Assistant

$7(4.0)$

RMN/ Staff nurse

139 (79.4)

Experience (Years)

$\begin{array}{ll}0-5 & 57(32.6) \\ 6-10 & 12(6.9) \\ 11-15 & 19(10.9) \\ 16-20 & 21(12.0) \\ 21-25 & 26(14.9) \\ 26-30 & 23(13.1) \\ 31-35 & 13(7.4) \\ 36+ & 4(2.3)\end{array}$

Mode of survey completion

$\begin{array}{lll}\text { Paper } & 38(21.7) & 0(0.0) \\ \text { Electronic } & 137(78.3) & 40(100.0)\end{array}$

Length of admission (months)

$\begin{array}{lll}0-3 & - & 14(35.0) \\ 3-6 & - & 9(22.5) \\ 6-12 & - & 6(15.0) \\ 12-24 & - & 3(7.5) \\ 24+ & - & 8(20.0)\end{array}$

This article is protected by copyright. All rights reserved. 


\section{Table 3: Principal components analysis for 17-item SHAS}

$\begin{array}{llc}\begin{array}{l}\text { Perceived } \\ \text { Manipulation }\end{array} & \begin{array}{c}\text { Perceived } \\ \text { Functionality }\end{array} & \begin{array}{c}\text { Freedom of } \\ \text { Choice }\end{array}\end{array}$

When individuals self-harm it is often to manipulate carers

People who self-harm are usually trying to get sympathy from others

0.88

A self-harming client is a person who is only trying to get attention

0.75

People who self-harm are typically trying to get even with someone

0.69

Acts of self-harm are a form of communication to their situation

Self-harming clients have a great need for acceptance and understanding

Self-harming individuals can learn new ways of coping

For some individuals self-harm can be a way of relieving tension

An individual has the right to self-harm

People should be allowed to self-harm in a safe environment

A rational person can self-harm

SHAS Self Harm Antipathy Scale Patterson et al. (2007)

This article is protected by copyright. All rights reserved. 
Table 4: Principal components analysis for 108-item ASc-Me

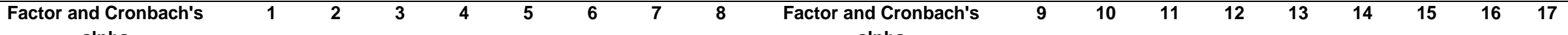
alpha

Constant observations*

$$
.93
$$

0.72

0.78

0.64

0.69

0.76

0.77

0.78

0.83

0.69

0.80

0.82

0.78

Care planning

.95

0.82
0.67
0.84

0.85

0.85
0.78

0.82

0.67

0.84

0.85

PRN with consent

.91
Refuse medical care

.95

Forced IM medication

.95

0.87

0.84

0.79

0.84

0.81

0.87

Therapeutic interventions

.95

0.72

0.81

0.77

0.76

0.80
0.80

Inappropriate medical care.

.96

This article is protected by copyright. All rights reserved. 
Active distractions

.93

0.80

0.94

0.91

0.92

Remain present during cutting

.95

0.89

0.89

0.84

0.88
Seclusion

.93

0.88

0.88

0.87

0.88

0.90

0.90

Informing other staff

.95

Providing sterile razors

.94

hysical restraint

This article is protected by copyright. All rights reserved. 
N.B. Columns labeled 2-17 are item loadings for a) Effectiveness; b) Acceptability; c) Respectfulness; d) Safety for staff; e) Safety for patients; f) Preparedness to use method (staff)/ Preparedness to be subject to measure (service users) relating to the corresponding factor. Column labeled 1 contains item loadings for two self-harm management methods ('observation in sight of' a-f and observation at arm's length' a-f); all 12-item loaded onto a single factor relabeled 'constant observations'. 
Table 5: Spearman's rho correlations between SHAS factors and selected harm-reduction related ASc-Me factors

\section{ASc-Me Factors}

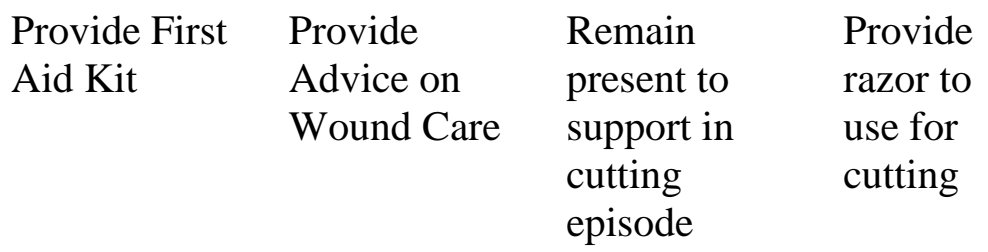

\section{SHAS-derived Factors}
1 Perceived Manipulation
0.07
0.04
0.13
$0.18^{*}$
2 Perceived Functionality
$0.24 * *$
$0.21 * *$
0.13
$0.22 * *$
3 Freedom of Choice
$0.36^{* *}$
$0.20 * *$
$0.15^{*}$
$0.51 * *$

$* \mathrm{P}<.05 ; * * \mathrm{P}<.01$

SHAS Self Harm Antipathy Scale Patterson et al. (2007)

ASc-Me Attitudes to Self-cutting Management Scale

This article is protected by copyright. All rights reserved. 
Table: 6: ASc-Me Staff/ service user mean self-cutting management technique scores and comparisons

\begin{tabular}{lllllll}
\hline Self-cutting management method & $\begin{array}{l}\text { Rank } \\
\text { Nursing staff }\end{array}$ & $\begin{array}{c}\boldsymbol{M}(\boldsymbol{S D}) \\
\text { Service } \text { users }\end{array}$ & $\begin{array}{l}\text { Rank } \\
\mathbf{t}(\boldsymbol{S D})\end{array}$ & $\begin{array}{l}\text { Independent Samples } \\
\text { (Cohen's } \boldsymbol{d})\end{array}$ \\
\hline Therapeutic interventions & 1 & $28.1(2.8)$ & 5 & $24.7(5.0)$ & $\mathrm{t}(213)=-5.82, P<0.001$ & $0.84(\mathrm{~N}>\mathrm{U})$ \\
Care planning & 2 & $28.0(2.8)$ & 1 & $25.9(3.5)$ & $\mathrm{t}(213)=-3.56, P=0.001$ & $0.67(\mathrm{~N}>\mathrm{U})$ \\
Suggest passive distraction techniques & 3 & $27.7(2.9)$ & $3=$ & $24.9(4.2)$ & $\mathrm{t}(213)=-3.96, P<0.001$ & $0.77(\mathrm{~N}>\mathrm{U})$ \\
Provide advice on wound care hygiene & 4 & $27.0(3.3)$ & 2 & $25.3(3.5)$ & $\mathrm{t}(213)=-2.92, P<0.01$ & $0.50(\mathrm{~N}>\mathrm{U})$ \\
Make other staff aware & 5 & $26.4(3.9)$ & 8 & $21.4(5.5)$ & $\mathrm{t}(213)=-6.65, P<0.001$ & $1.03(\mathrm{~N}>\mathrm{U})$ \\
Suggest active distractions & 6 & $25.7(4.5)$ & 7 & $22.1(4.7)$ & $\mathrm{t}(213)=-4.50, P<0.001$ & $0.80(\mathrm{~N}>\mathrm{U})$ \\
Provide first aid kit & 7 & $25.5(3.7)$ & 6 & $23.6(4.5)$ & $\mathrm{t}(213)=-2.79, P<0.01$ & $0.46(\mathrm{~N}>\mathrm{U})$ \\
Offer PRN & 8 & $24.3(4.3)$ & $3=$ & $24.9(4.4)$ & $\mathrm{t}(213)=.836, \mathrm{NS}$ & - \\
Change observation status to 'constant' & 9 & $20.5(5.0)$ & 10 & $19.4(5.3)$ & $\mathrm{t}(213)=-1.15, P=\mathrm{NS}$ & - \\
Change observations to 'intermittent' & 10 & $19.5(5.6)$ & 9 & $21.3(5.1)$ & $\mathrm{t}(213)=1.903, P=\mathrm{NS}$ & - \\
Remain present & 11 & $18.5(5.8)$ & 11 & $18.3(6.2)$ & $\mathrm{t}(213)=-.16, P=\mathrm{NS}$ & - \\
Provide sterile razor & 12 & $18.4(5.6)$ & 12 & $18.0(6.2)$ & $\mathrm{t}(213)=-.35, P=\mathrm{NS}$ & - \\
Seclusion & 13 & $18.2(6.6)$ & 13 & $15.3(5.8)$ & $\mathrm{t}(213)=-2.51, P<0.05$ & $0.46(\mathrm{~N}>\mathrm{U})$ \\
Physical restraint & 14 & $14.5(5.8)$ & 15 & $11.6(5.7)$ & $\mathrm{t}(213)=-2.84, P<0.01$ & $0.50(\mathrm{~N}>\mathrm{U})$ \\
Forced Intramuscular Medication & 15 & $13.0(6.2)$ & 14 & $12.3(5.2)$ & $\mathrm{t}(213)=-.66, P=\mathrm{NS}$ & - \\
Refuse treatment & 16 & $8.7(4.2)$ & 16 & $10.2(5.1)$ & $\mathrm{t}(213)=2.04, P<0.05$ & $0.33(\mathrm{~N}<\mathrm{U})$ \\
Inappropriate treatment & 17 & $8.5(4.0)$ & 17 & $8.0(2.5)$ & $\mathrm{t}(213)=-.790, P=\mathrm{NS}$ & - \\
\hline
\end{tabular}

N Nurse; U Service User. NS Not significant. N.B., Higher score denotes greater approval of the technique

This article is protected by copyright. All rights reserved. 


\section{ATTITUDES TO SELF-CUTTING MANAGEMENT SCALE (ASC-ME)}

\section{Self-cutting management technique or intervention}

Please indicate in the right hand column the extent to which you agree or disagree that each technique is acceptable overall in safely and effectively helping the person who self-cuts or is experiencing urges to self-cut while respecting their autonomy and dignity

\section{Passive distractions [Suggest and provide indirect alternatives to} cutting - distraction techniques, relaxation techniques, engaging in unrelated activities]

\section{Active distractions [Suggest/ provide pinging elastic bands, using} ice cubes, drawing on self with red pen, toothbrush on skin]

\section{Care planning [Develop a person-cent red, individualised care plan} and risk assessment to address cutting]

\section{Therapeutic interventions [Provide psychological interventions} aimed at self-harm reduction e.g. problem solving approaches, consideration of self-cutting function]

\section{Wound care hygiene [Provide advice on wound care, cleaning,} and signs of infection]

\section{Make others aware [Make all nursing staff aware that a person is} self-cutting or feels like self-cutting]

\section{First Aid kit [Provision of a First Aid kit containing dressings, etc. for} immediate wound care]

\section{Sterile self-cutting equipment [Provide sterile cutting} implements - razors or scalpels - to lower risk of infection following a cutting event]

\section{Maintain nursing presence [Nurse remains present during self- cutting event to provide support and ensure safety]}

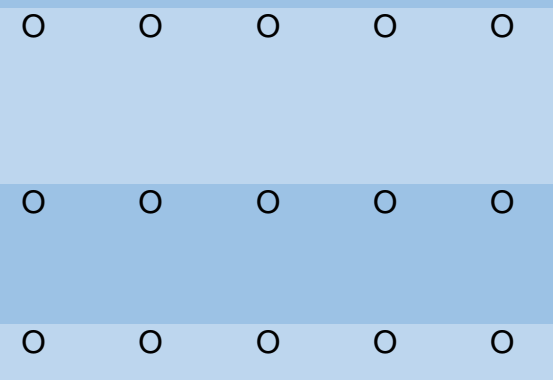

This article is protected by copyright. All rights reserved. 
with patient consent]

11.Intermittent observation [Increase observations so patient is

$\begin{array}{lllll}0 & 0 & 0 & 0 & 0\end{array}$

checked on at predetermined intermittent times by staff e.g. every 10 minutes]

12. Close observation [Increase observations so patient remains in

$\begin{array}{lllll}0 & 0 & 0 & 0 & 0\end{array}$

sight of/ within arm's length of a member of nursing staff at all times]

13.Seclusion [Moving and isolating patient within their room or another

$\begin{array}{lllll}0 & 0 & 0 & 0 & 0\end{array}$ specified area of the ward]

14.Inappropriate treatment [e.g., stitch wounds without

$\begin{array}{lllll}0 & 0 & 0 & 0 & 0\end{array}$

anaesthetic as a disincentive to self-cutting]

15.Refuse treatment [ Refuse to provide treatment to the individual

$\begin{array}{lllll}0 & 0 & 0 & 0 & 0\end{array}$

who engages in self-cutting]

16.Physical restraint [Prevent patient moving/self-cutting by nursing

staff using specific manual holding techniques]

17.Forced IM medication [Forced Intra Muscular medication -

$\begin{array}{lllll}0 & 0 & 0 & 0 & 0\end{array}$ injection of intramuscular medication (e.g. sedatives) given without consent ]

Please do not amend this scale without permission.

This article is protected by copyright. All rights reserved. 